\title{
Interference Mitigation in Satellite Personal Communication Networks Using Adaptive Antenna Arrays and Filtering Technique
}

\author{
Sunday E. Iwasokun ${ }^{1}$, Michael O. Kolawole ${ }^{2}$ \\ ${ }^{1}$ Department of Electrical and Electronics Engineering, School of Engineering and Engineering Technology, Federal University of \\ Technology, Akure, Nigeria. \\ Email: \{wasokunday, kolawolm\}@yahoo.com
}

Received April 27 $7^{\text {th }}, 2010$; revised August $3^{\text {rd }}, 2010$; accepted August $4^{\text {th }}, 2010$.

\begin{abstract}
We address the problem of interference as related to Satellite Personal Communication Networks (S-PCNs). Basic low Earth orbit (LEO) constellation is considered. The paper uses combined adaptive antenna arrays and adaptive filtering technique. This hybrid linear adaptive technique provides improved performance eliminating interference, particularly uncorrelated signals residing in the antenna sidelobes.
\end{abstract}

Keywords: S-PCN, LEO, Adaptive Array Antennas, Adaptive Filtering, Interference Elimination

\section{Introduction}

A Satellite personal communication network (S-PCN) provides universal access to a wide range of services and across transnational boundaries. It faces multitude of challenges particularly those related to user terminals, the space segment, regulatory challenges, [1], as well as technical issues including link diversity, traffic allocation [2], quality of desired service (QoS) and the associated grade of service (GoS) [3], etc. The interference encroachment into the signals received by systems terminals impacts on the QoS and GoS as well, and importantly the network congruous scope to deal with the motion of the satellite as well as that of the mobile user [4]. Most mobile stations' transceivers signals are ideally circularly polarized and isotropic in nature, with a single low gain antenna element. However, these single element units are particularly more susceptible to noise interference because they receive signals from all directions. Mounting adaptive antenna arrays on the mobile unit is an approach suitable to combating interference problem as communication satellite traverses the sky, which this study attempts to investigate. Of particular concern is the interference on voice channels causing crosstalk; e.g. where the subscriber hears interference in the background. Figure 1 demonstrates an S-PCN user affected by random, multidimensional interferences.

\section{Array Geometry}

An adaptive array antenna unit is designed, as in Figure 2, bringing all the signals received by the various elements from a particular source into phase for further processing, as well as achieving some desired performance, such as maximizing the received signal-to-noise ratio (SNR). Drawing from [5] and [6], we analyse the antenna array assuming that 1) there is a direct path for the signal from the satellite and interfering signal is uncorrelated with the desired signal, 2) all the array elements are placed closely enough such that there is no significant amplitude

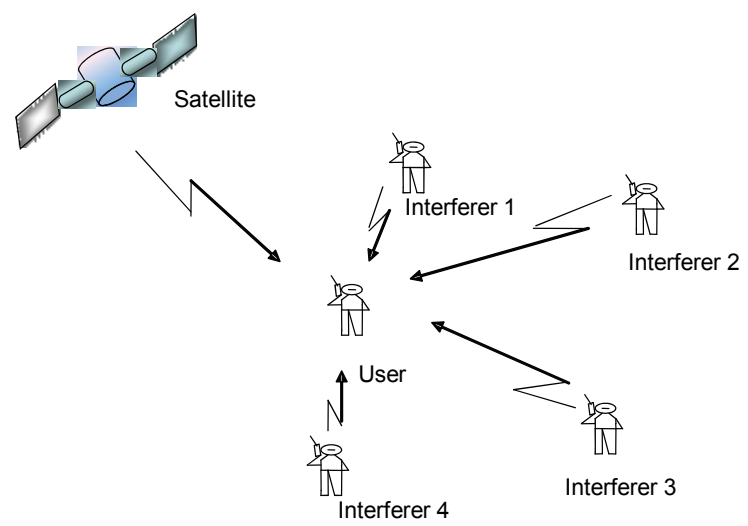

Figure 1. PCN mobile user affected by interference. 


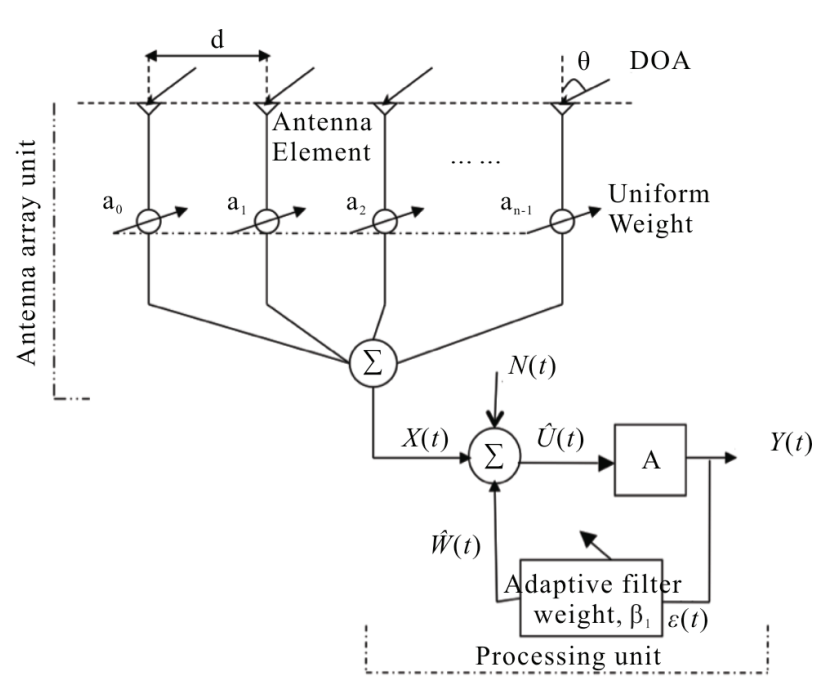

Figure 2. An adaptive antenna and adaptive filter for $S$ PCN mobile terminal.

variation due to the difference in propagation path length for any two elements, and 3 ) there is no significant difference in $\theta$ - the direction-of-arrival (DOA) - of a particular plane wave at any two elements.

For $\mathrm{N}$ elements array of identical characteristics, the first pulse is taken as the phase centre, $f(\tau)$. The second pulse is advanced by $\tau$ and denoted by $f(t-\tau)$, the third pulse is advanced by $2 \tau$ and denoted by $f(t-2 \tau)$, and so on. For convenience, five elements are used, so

$$
\begin{aligned}
f_{T}(t)= & a_{o} f(t)+a_{1} f(t-\tau)+a_{2} f(t-2 \tau)+ \\
& a_{3} f(t-3 \tau)+a_{4} f(t-4 \tau)
\end{aligned}
$$

where $a_{0}, a_{1} \ldots a_{5}$ are the weights/gains of each element of the array. In adaptive antenna array, the weights/gains of each element are taken to be the same (i.e., $a_{0}=$ $a_{1}=\ldots=a_{4}$ ), conforming to practical situation, and the elements to be equally spaced. Had the weights variable, there is possibility they may modulate the desired signal. Now, if the first pulse is Fourier transformed and is represented by $f_{\Delta}(\omega)$, then

$$
\begin{aligned}
f_{T}(\omega)= & a_{0} f_{\Delta}(\omega)+a_{1} f_{\Delta}(\omega) e^{-j \omega \tau}+a_{2} f_{\Delta}(\omega) e^{-j 2 \omega \tau}+ \\
& a_{3} f_{\Delta}(\omega) e^{-j 3 \omega \tau}+a_{4} f_{\Delta}(\omega) e^{-j 4 \omega \tau}
\end{aligned}
$$

By multiplying (2) by $e^{-j \omega \tau}$ and then subtracting the resulting expression from (1), and rearranging ensuing expression and using known geometric series expansion we have

$$
f_{\tau}(\omega)=a_{0} f_{\Delta}(\omega)\left[e^{-j 2 \omega \tau}\left(\frac{\sin (5 \pi f \tau)}{\sin (\pi f \tau)}\right)\right]
$$

Typical S-PCN frequencies of 1.376 and $1.80 \mathrm{GHz}$ $[6,7]$ are used to examine the antenna array's behaviour using (3). Resulting graphs are shown in Figure 3. The sidelobes performance has an important impact on inter-

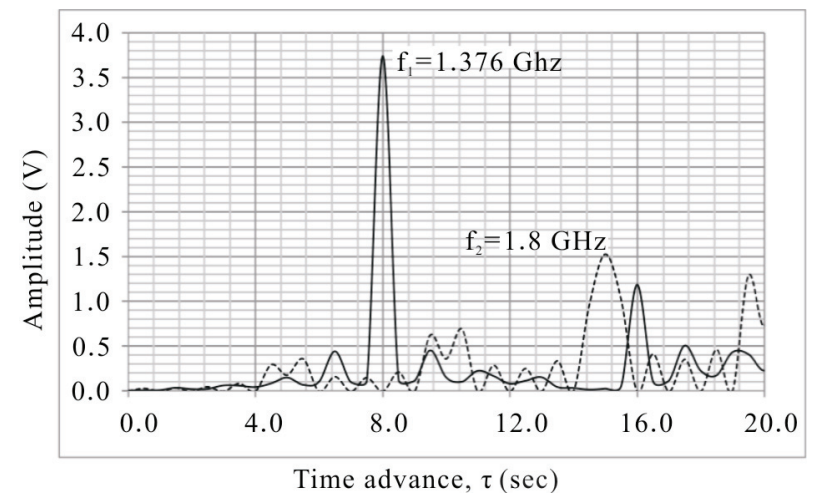

Figure 3. Antenna array's response at typical S-PCN frequencies.

ference received from adjacent satellites (and ground based signals operating on same bands), as well as in determining antenna noise temperature. To successfully eliminate, or reduce significantly, the effect of external interference, the sidelobes have to be attenuated, if not removed. The sidelobes are cancelled or removed in this paper by the adaptive array process.

\section{Processing Unit: Modelling Interference with Adaptive Filter}

Our approach models the effect of interferers on the S-PCN systems using interferences as recursive random processes, $N_{i}(t)$ with the array antenna. The input to the processor in Figure $\mathbf{2}$ is the inverse Fourier $\mathfrak{J}^{-1}\left[f_{\tau}(\omega)\right]$ of the array output, which now becomes $X(t)$ to the summer. The effect of noise from each antenna element is factored in at the output; that is, for $n$ elements with equal average signal-to-noise (SNR), the average SNR of the array combiner is $10 \log _{10}(n)$, which is higher in $\mathrm{dB}$ than the average SNR of any one of the elements [8].

Following Figure 2, the recursive-filtering algorithm can be represented as a linear, discrete-time (t) model of the form:

$$
\begin{aligned}
& \hat{U}(t)=X(t)+N(t)-\hat{W}(t) \\
& Y(t)=A \hat{U}(t)
\end{aligned}
$$

where $A(t)$ is the system parameter; $\hat{W}(t)$ is optimum adaptive but weighted filter and $N(t)$ is additive, uncorrelated system interference, assumed white, zeromean Gaussian and stochastic; and $Y(t)$ filter (or measurement) output. The optimum interference $\hat{W}(t)$ is defined as

$$
\hat{W}(t)=\sum_{i=0}^{k} \beta_{i}(t) N_{t h}(t-i)
$$

where $N_{t h}$ is the acceptable interference threshold, and $k$ is the filter order. The proceeding coefficient of the filter 


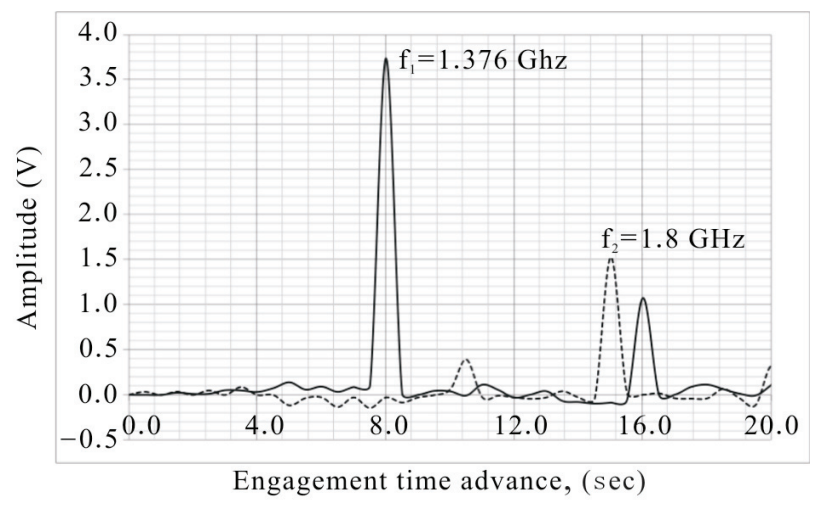

Figure 4. Simulated received response of mobile terminal at typical S-PCN frequencies.

can be estimated from the present coefficient and other thresholds:

$$
\beta_{i}(t+1)=\beta_{i}(t)+2 \eta X(t) N_{t h}(t-i)
$$

where $\eta$ is the convergence constant.

The adaptive filter adapts the filter coefficients to achieve desired signal ensuring convergence; that is, minimizing error $\varepsilon(t)$ at each time index:

$$
\varepsilon(t)=Y(t)-A \hat{U}(t)
$$

Ensuring fast convergence a local minimum is sought leading to establishing threshold value; i.e.,

$$
\xi(t)=\sum_{j=0}^{k}\left|\beta_{t}(j)-\beta_{t-1}(j)\right| \leq \alpha_{t h}
$$

where $\alpha_{t h}$ local minimum threshold. The adaptation gain $g(m)$ is introduced for coefficient updating recursion for the period of the signal measurement:

$$
g(m)=\left|\sum_{i=0}^{m} \frac{\hat{U}(i)}{X(i)+N(i)}\right|
$$

where $m$ is the period which the mobile terminal engages the network.

As shown in Figure 4, application of adaptive filter in conjunction with the antenna array processing shows encouraging responses in terms of interference reduction.

The implication of our method is that the variation of the filter's weights, as a result of movement of the mobile users, may affect the effectiveness of the system as the satellites move from orbit to another; low earth orbit (LEO) through to geostationary orbit (GEO), for example [9]. However, a mobile user of any of the systems might experience the same problem since the environment is changing too rapidly for the weights to converge. This is an area that is being investigated. Also, the relative motion between the user and the satellite reference may be another source of error resulting in Doppler shift. Recent work of [10] suggests that using frequency lock loop could compensate for this error. Whilst our technique has utilised uniform weight for the antenna array, this should not a problem if variable weights were to be utilised [11]. Moreover, since some of the satellite constellations utilize code division multiple access (CDMA) as the multiple access technique, pseudorandom noise codes can also be utilized to monitor the adaptive array weights.

\section{Conclusions}

We have presented an antenna-array plus adaptive filtering model as a way of eliminating the effect of interference in satellite personal communication systems. This technique has shown that it can eliminate an uncorrelated signals residing in the antenna sidelobes successfully. This technique is easily adaptable to S-PCN in LEO operational environment as a result of shorter time required by LEO satellites to move across the sky.

\section{REFERENCES}

[1] L. Ghedia, "Satellite Personal Communication Networks," In: B. G. Evans, Ed., Satellite Communication Systems, Institution of Electrical Engineers, 1999.

[2] R. Septiawan, "Multiservice Traffic Allocation in LEO Satellite Communications," Ph.D. Dissertation, Bond University, Gold Coast, 2004.

[3] R. Conte, "Satellite Rural Communications: Telephony and Narrowband Networks," International Journal of Satellite Communications and Networking, Vol. 23, No. 2, 2005, pp. 307-321.

[4] M. O. Kolawole, "A Course in Telecommunication Engineering," S Chand, New Delhi, 2009.

[5] M. I. Skolnik, "Introduction to Radar Systems," McGraw-Hill, Singapore, 1981.

[6] M. O. Kolawole, "Radar Systems, Peak Detection and Tracking," Elsevier, Oxford, 2003.

[7] E. Lutz, "Issues in Satellite Personal Communication Systems," Wireless Networks, Vol. 4, No. 2, 1998, pp. 109-124.

[8] M. A. Ingram, R. Romanofsky, R. Q. Lee, F. Miranda, Z. Popovic, J. Langley, W. C. Barott, M. U. Ahmed and D. Mandi, "Optimizing Satellite Communications with Adaptive and Phased Array Antennas," Earth Science and Technology Conference, Crowne Plaza, 2004, pp. $1-7$.

[9] S. E. Iwasokun and M. O. Kolawole, "Using Hybrid Adaptive Techniques to Reduce Multipath Effects in S-PCN Mobile Terminals," International Journals of Communications, Network and System Sciences, 2010 (In press).

[10] M. Rao, L. L. Presti, M. Fantino and G. Garbo, "A Soft- 
ware Receiver Adaptive Phase Lock Loop Method," International Global Navigation Satellite Systems Society (IGNSS) Symposium, Sydney, December 2009, pp. 1-13.

[11] S. E. Iwasokun, "Elimination of Interference and Multipath Signals in Satellite Personal Communication Sys- tems Using Adaptive Antenna Array," M. Eng. Thesis, Federal University of Technology, Akure, 2010. 